\title{
Association of peripheral nerve blocks with postoperative outcomes in ambulatory shoulder surgery patients: a single-centre matched-cohort study Association entre les blocs nerveux périphériques et des critères
d'évaluation postopératoires chez des patients ambulatoires
opérés de l'épaule: étude de cohorte appariée monocentrique
}

Gavin M. Hamilton, MD, MSc $\mathbb{D} \cdot$ Reva Ramlogan, MD, BSc • Anne Lui, MD, MSc • Colin J. L. McCartney, MB, ChB, PhD • Faraj Abdallah, MD, BSc •

Daniel I. McIsaac, MD, MPH

Received: 29 April 2018/Revised: 24 July 2018/Accepted: 24 July 2018/Published online: 17 October 2018

(C) Canadian Anesthesiologists' Society 2018

\begin{abstract}
Purpose Regional anesthesia may have immediate postoperative advantages compared with general anesthesia, but its impact on post-discharge outcomes is poorly described. Our objective was to measure the association between regional anesthesia and outcomes after ambulatory shoulder surgery.
\end{abstract}

Electronic supplementary material The online version of this article (https://doi.org/10.1007/s12630-018-1234-8) contains supplementary material, which is available to authorized users.

G. M. Hamilton, MD, MSc

Department of Anesthesiology and Pain Medicine, University of Ottawa, 1053 Carling Ave, Ottawa, ON K1Y 4E9, Canada

R. Ramlogan, MD, BSc · A. Lui, MD, MSc

C. J. L. McCartney, MB, ChB, PhD · F. Abdallah, MD, BSc Department of Anesthesiology and Pain Medicine, University of Ottawa, 1053 Carling Ave, Ottawa, ON K1Y 4E9, Canada

The Ottawa Hospital Research Institute, University of Ottawa, Ottawa, ON, Canada

D. I. McIsaac, MD, MPH ( $)$

Department of Anesthesiology and Pain Medicine, University of Ottawa, 1053 Carling Ave, Ottawa, ON K1Y 4E9, Canada e-mail: dmcisaac@toh.ca

The Ottawa Hospital Research Institute, University of Ottawa, Ottawa, ON, Canada

School of Epidemiology and Public Health, University of Ottawa, Ottawa, ON, Canada
Methods We conducted a historical cohort study at The Ottawa Hospital. Adults $\geq 18$ yr old having elective ambulatory shoulder surgery from January 1, 2011 to December 31, 2016 were included. Using one-to-many coarsened exact matching (CEM) to adjust for potential confounders, we matched peripheral nerve block (PNB) patients to those without a PNB. Within the matched cohort, we assessed whether PNBs were associated with our primary composite outcomes, comprising unplanned admissions, emergency department visits, readmissions, or death within 30 days of surgery.

Results There were 1,623 patients who met the inclusion criteria; PNBs were placed in 1,382 (85.2\%) patients. CEM matched 211 patients who did not receive a PNB to 950 patients with similar characteristics who did receive a $P N B(n=1,161 ; 71.5 \%$ of total cohort). In patients who received a PNB compared with those who had no PNB, there was no difference in risk of composite outcome (relative risk, 1.58; 95\% confidence interval [CI], 0.83 to 3.01), or hospital costs (ratio of means 0.73; 95\% CI, 0.21 to 2.49).

Conclusion Peripheral nerve blocks in ambulatory shoulder surgery were not associated with a significant difference in a composite of adverse postoperative outcomes. Nevertheless, given the lower than expected incidence and moderate effect size associating PNBs with post-discharge events, future large prospective trials are needed to assess post-discharge outcomes.

Trial registration www.clinicaltrials.gov (NCT03309 644). Registered 13 October 2017. 


\section{Résumé}

Objectif L'anesthésie régionale peut présenter des avantages postopératoires immédiats par rapport à une anesthésie générale, mais ses répercussions sur l'évolution après le congé sont mal connues. Notre objectif était de mesurer l'association entre l'anesthésie régionale et l'évolution des patients après une chirurgie ambulatoire de l'épaule.

Méthodes Nous avons mené une étude de cohorte historique à l'hôpital d'Ottawa. Des adultes âgés de 18 ans et plus ayant subi une chirurgie ambulatoire de l'épaule entre le $1^{\text {er }}$ janvier 2011 et le 31 décembre 2016 ont été inclus. Utilisant la technique d'appariement CEM (coarsened exact matching) "d'un-à-plusieurs » pour tenir compte des facteurs confondants éventuels, nous avons apparié des patients ayant subi un bloc nerveux périphérique $(B N P)$ à des patients sans BNP. Au sein de la cohorte appariée, nous avons évalué si les BNP étaient associés à notre critère d'évaluation composite principal, comprenant les hospitalisations non planifiées, les visites aux urgences, les réhospitalisations ou les décès dans les 30 jours suivant la chirurgie.

Résultats $A u$ total, 1623 patients satisfaisaient les critères d'inclusion; des BNP ont été réalisés chez 1382 patients (85,2\%). Le logiciel de CEM a apparié 211 patients n'ayant pas reçu de BNP à 950 patients présentant des caractéristiques similaires et ayant reçu un $B N P(n=1161 ; 71,5 \%$ de la cohorte totale). Chez les patients ayant reçu un BNP comparés aux patients sans $B N P$, aucune différence n'a été observée sur le risque du critère composite (risque relatif, 1,58; intervalle de confiance [IC] à $95 \%: 0,83$ à 3,01) ou sur les coûts hospitaliers (rapport des moyennes 0,73; IC à $95 \%, 0,21$ à 2,49).

Conclusion Les blocs nerveux périphériques n'ont pas été associés à une différence significative pour un critère d'évaluation composite d'événements indésirables postopératoires dans la chirurgie ambulatoire de l'épaule. Néanmoins, compte tenu de l'incidence plus faible que prévue et de la taille d'effet modéré associant les BNP aux événements post congé, de futurs grands essais prospectifs seront nécessaires pour évaluer les complications post congé.

Enregistrement de l'essai clinique $w w w$.ClinicalTrials. gov (NCT03309644). Enregistré 13 octobre 2017.

Ambulatory surgeries are increasingly common. ${ }^{1,2}$ The cost-effectiveness and efficiency of ambulatory surgery depends on the ability to discharge patients to their previous residence on the day of surgery. Over $25 \%$ of unanticipated admissions after ambulatory surgery are attributable to anesthesiology-based care and interventions. $^{3} \quad$ Furthermore, shoulder surgery is associated with a three-fold increase in unanticipated hospital admissions compared with other orthopedic ambulatory surgeries. ${ }^{4}$ Specific reasons for this association are unclear. Nevertheless, since shoulder surgery is one of the most painful common surgeries, ${ }^{3,5}$ anesthesiology care may be particularly important in influencing patient-centred and health system outcomes.

There are variations in perioperative anesthesia care for shoulder surgery. This likely reflects a lack of evidence on the comparative effectiveness of the different approaches to guide the choice of optimal perioperative management strategies. ${ }^{6}$ The majority of ambulatory shoulder surgeries are performed with general anesthesia (GA), and there is wide variation (20-86\%) in peripheral nerve block (PNB) administration in reported institutional anesthesia practices. $^{2,7}$ No studies have evaluated post-discharge outcomes following ambulatory shoulder surgery and regional anesthesia in Canada.

Our objective was to measure the association of interscalene brachial plexus catheters with outcomes of ambulatory shoulder surgery at a Canadian academic health sciences centre. Because the association between receipt of a PNB and outcomes may be impacted by confounding and indication biases, we utilized coarsened exact matching (CEM) - a novel matching technique, which may decrease bias relative to propensity scores (PS) - to match PNB patients to non-PNB patients in a fashion where all measured covariates are exactly the same within each match. We hypothesized that patients receiving a PNB would have a lower rate of composite primary outcome, which included unplanned day-of-surgery admissions, and emergency department (ED) visits or hospital readmission within 30 days of surgery. We further hypothesized that PNBs would decrease the incidence of each of the component outcomes, as well as postoperative costs.

\section{Methods}

Setting

After ethical approval was received on 19 October, 2016 from the Ottawa Health Sciences Network Research Ethics Board (REB \#: 20160800-01H), we conducted a historical cohort study at The Ottawa Hospital (TOH). TOH is an academic health sciences centre with two inpatient campuses and one free standing ambulatory surgery centre. Ambulatory surgeries are performed on all campuses. 
At $\mathrm{TOH}$, patients scheduled for shoulder repair or arthroplasty have access to our ambulatory regional anesthesia program, which includes continuous outpatient perineural infusion catheters. This program was developed with consensus from participating surgeons and anesthesiologists, and receives both resource and facility support from hospital administration. Patients receive information on the nerve block in our preassessment clinic. This education is reinforced with formalized postoperative care instructions provided by nurses in the postanesthesia care unit (PACU). Any patient who consents to the PNB is provided with verbal and written instructions for postoperative care and emergency contact numbers.

The interscalene brachial plexus single shot block, or catheter, is performed using an aseptic technique and minimal sedation. Under dynamic ultrasound guidance, $20-40 \mathrm{~mL}$ of $0.5 \% \quad\left(5 \mathrm{mg} \cdot \mathrm{mL}^{-1}\right)$ ropivacaine with $1: 400,000\left(2.5 \mu \mathrm{g} \cdot \mathrm{mL}^{-1}\right)$ epinephrine is injected through the needle or catheter prior to GA or surgery. In addition to the PNB, patients receive either GA with volatile anesthetics or intravenous sedation with propofol. Patients with a catheter are discharged with a continuous infusion of $0.2 \%\left(2 \mathrm{mg} \cdot \mathrm{mL}^{-1}\right)$ ropivacaine at a rate of 5 $\mathrm{mL} \cdot \mathrm{hr}^{-1}$ with a patient-controlled bolus option of $5 \mathrm{~mL}$ every $30 \mathrm{~min}$. The anesthesiologist who performed the PNB follows up with the patient by telephone contact until the infusion is complete and the catheter is removed.

For those patients not receiving a PNB, there was no set protocol at our institution for the GA or postoperative analgesic regimen. The general practice at $\mathrm{TOH}$ is that prior to emergence from GA, the surgical team infiltrates $0.25 \%$ bupivacaine with 1:200000 epinephrine (with no additional nonsteroidal anti-inflammatory or opioid in the solution) around the surgical field. The patient is discharged home and the surgeon determines the postoperative analgesic regimen. It is also common practice to advise patients to use foundational analgesia (i.e., acetaminophen and/or nonsteroidal antiinflammatory), in addition to an opioid.

\section{Data sources}

Data were collected from the Ottawa Hospital Data Warehouse (OHDW), a peer-reviewed central data repository that stores a combination of administrative and clinical data for all patients cared for at TOH. For this study, OHDW databases accessed included the Canadian Institute for Health Information (CIHI) National Ambulatory Care Reporting System (NACRS), which records all ambulatory surgical procedures performed, present on admission diagnoses, and ED visits; the CIHI Discharge Abstract Database (DAD), which records all inpatient hospital admissions and present on admission diagnoses; and the Surgical Information Management System (SIMS), which is the legal standard medical record for all surgical procedures and anesthesia care provided at $\mathrm{TOH}$. The analytic dataset was created by a trained OHDW data analyst who was independent from the study team. The analysis was performed by the lead author and was overseen by the senior author. This study follows the Strengthening the Reporting of OBservational studies in Epidemiology and the REporting of studies Conducted using Observational Routinely collected health Data guidelines. $^{8,9}$

\section{Design and study population}

Our study population included patients aged $18 \mathrm{yr}$ and older, who underwent an elective ambulatory shoulder surgery at $\mathrm{TOH}$. Participants were identified through the NACRS using previously studied Canadian Classification of Interventions (CCI) codes to identify the following shoulder surgeries: shoulder arthroscopy, arthroplasty synthetic repair, implantation of an internal device shoulder joint, repair of recurrent dislocation, other shoulder repair, shoulder arthrotomy, division of joint capsule, lining or cartilage shoulder, synovectomy shoulder, other local excision, destruction of shoulder joint, or other joint excision (shoulder) (Appendix A, available as Electronic Supplementary Material [ESM]). ${ }^{10}$

We compiled a patient-level analysis cohort by including only the first surgery for any participant in the study period. Our start time of January 1, 2011 was chosen to coincide with the system-wide introduction of SIMS. December 31, 2016 was chosen as the end point since this is the latest time at which all datasets were complete.

\section{Exposure}

Our primary exposure was the anesthesia type provided for ambulatory shoulder surgery. This was determined from SIMS, where an anesthesia type must be entered for all cases and where a standardized checklist for technical description of PNBs is entered for all cases involving regional anesthesia. We coded our primary exposure as a binary variable. Patients who had a GA with no PNB were coded as $\mathrm{PNB}=0$. Patients who had a documented PNB were coded as $\mathrm{PNB}=1$. Patients receiving a PNB may have received a concurrent GA or only sedation.

We compared isolated GA (iGA) with PNB ( \pm GA). For sensitivity analysis, we further subcategorized the PNB group into GA vs sedation, using iGA as our reference category. This was repeated within a subset of patients who had a continuous catheter inserted instead of a single shot block, to allow for further sensitivity analysis within the 
group of patients who received a PNB. Further sensitivity analysis was preformed to assess the effect of procedure type (open $v s$ arthroscopic).

\section{Outcomes}

Our primary outcome was a composite that included unplanned admissions on the day of surgery (from NACRS), post-discharge ED visits within 30 days of surgery (from NACRS), unplanned readmission within 30 days of surgery (any non-elective admission from the DAD), and death from any cause (Appendix B, available as ESM). Mortality was included in the composite measure because, even though it is a rare after ambulatory surgery, it is a competing risk. ${ }^{11}$ Thirty-day outcome windows are the standard reporting interval for most postoperative outcomes, and are routinely monitored at TOH. Our secondary outcome was health system costs incurred by $\mathrm{TOH}$ in the 30 days after completion of the surgery (not including the cost of the anesthetic and surgery itself). Costs were calculated using standardized methods and included both direct and indirect costs standardized to 2016 Canadian dollars. This method accounts for an individual patient's resource intensity weight, their case mix group, as well as fixed and indirect costs to the hospital based on the patient's location of care. ${ }^{12}$

\section{Covariates}

Patient demographics, comorbidities, campus, and surgery type may confound the association between receipt of a PNB and outcomes. In addition, year of surgery may also bias this association as care processes and outcome rates tend to change over time. Therefore, we collected baseline data on all participants including age, sex, Elixhauser comorbidity index ${ }^{13}$ American Society of Anesthesiology physical status (ASA-PS) score, and the year and type of surgery based on the alpha-numeric CCI code recorded in NACRS. Specific identification and definition of our confounding variables are described in Appendix C (available as ESM).

\section{Data analysis}

The dataset was created and manipulated using SAS version 9.4 (SAS Institute, Cary, NC, USA). Stata Statistical Software v10 (College Station, StataCorp LP, TX, USA) was used for all analyses. Descriptive statistics with absolute standardized differences were used to describe characteristics between exposure groups. Standardized differences of $10 \%$ or less are considered to represent an adequate balance of covariates. ${ }^{14}$
The unadjusted association between PNB and outcomes was analyzed. To account for differences between groups, we utilized CEM prior to our adjusted analysis to create a matched cohort. ${ }^{15}$ Coarsened exact matching is a novel type of monotonic imbalance bounding, which is used to preprocess the data. Contrary to PS methods, which match exposed and non-exposed participants on a summary score (even though, for example, they have had different procedures, or have different comorbidity burdens), CEM matches participants exactly on each specified variable. Coarsening refers to the fact that one may set a range for acceptable matching within a continuous variable (for example, within ten years of age). Coarsened exact matching greatly decreases model-dependence, requires fewer assumptions, and, most importantly, lowers bias (by removing heterogeneity) relative to PS matching. ${ }^{16,17}$ Our CEM model included patient sex (binary), age (categorical; $<40,40-65,>65 \mathrm{yr}$ ), year of surgery (categorical; using biennial groupings), ASA-PS (binary; $\leq 2,>2$ ), campus, anatomical location of intervention (CCI three-digit code; shoulder joint, rotator cuff, shoulder muscle), surgery type (open $v s$ arthroscopic) and Elixhauser comorbidity index (as binary variables using previously published data). ${ }^{18}$ Our primary analysis was conducted in a one-to-many matching method as per defined methods. ${ }^{15}$

For our binary outcomes, the unadjusted and CEMadjusted analyses measured the association between a PNB and our composite outcome using chi-square tests. Risk ratios (RR) and absolute risk differences, along with $95 \%$ confidence intervals (CI), were generated. For cost analyses, we utilized a generalized linear model with a gamma response distribution, as this has been shown to reduce bias and error in studies of surgical costs, which tend to be skewed. ${ }^{19}$ Costs attributable to the presence of a block were estimated by calculating the predicted cost for each participant using a generalized linear model with a log link and gamma response distribution, which included the same covariates as the CEM match algorithm. Two hundred bootstrap samples were then created using a one-to-one sampling rate to estimate the mean attributable cost, along with non-parametric $95 \%$ CI by sampling the median, 2.5th, and 97.5th percentiles of the bootstrap distribution of mean cost between those with and without a block.

To determine whether costs were simply a function of the components of our primary outcome we created an ordinary least squares linear regression model to estimate the proportion of variation in costs attributable to the component outcomes. Cost was the dependent variable and each part of the composite outcome was entered as a binary explanatory variable to estimate the model's $\mathrm{R}^{2}$ value. 
Sensitivity analyses

We performed several pre-specified analyses to test the robustness of our primary analysis. First, we conducted CEM analysis with a one-to-many match (which tends to improve precision compared with one-to-one matching). ${ }^{20}$ Because matched cohorts provide an estimate of the average treatment effect in the treated, but inevitably exclude individuals from the analysis because they go unmatched, we also performed traditional regression analysis, which provides an average treatment effect (i.e., what would happen if we moved all individuals from not having a PNB to having one). ${ }^{21}$ We used a generalized linear model with a log link and binomial response distribution (i.e., $\log$ binomial regression) for this analysis. Log binomial regression estimates a RR (as opposed to logistic regression, which estimates an odds ratio), which was the measure used in our primary analysis. ${ }^{22} \mathrm{We}$ also performed a separate analysis for each component of the composite outcome within the CEM one-to-many matched cohort. Finally, to account for coarsening applied to categorical or continuous variables in the CEM matching algorithm (age, ASA-PS score, year, Elixhauser index), we performed a multivariable log binomial regression analysis within the CEM one-tomany matched cohort for the primary composite outcome with age and Elixhauser index included as continuous variables, year as a categorical variable, and ASA score as an ordinal variable with ASA I as the reference. To assess the impact of PNB catheters, we compared the subset of patients who had a continuous catheter instead of a single shot block using log binomial regression.

\section{Sample size}

Readmission and ED rates vary substantially between studies of ambulatory shoulder surgery. ${ }^{23-26}$ Studies of United States data reported an average unplanned admission rate of $13.5 \%$, while Canadian ambulatory surgery data report a $>10 \%$ rate of post-discharge ED visits or readmissions. Therefore, prior to cutting our data, we estimated a conservative primary outcome rate of $15 \%$. Based on this estimate, a sample size of 239 participants per arm, with a significance level of 0.05 , would provide $80 \%$ power to detect a $50 \%$ decrease in outcome between the PNB and no PNB groups; 350 participants per arm would provide $90 \%$ power. Nevertheless, we included all available participants as data were readily available.

\section{Missing data}

Main outcome and exposure variables were complete for all participants. The ASA-PS score was missing for six participants but all other outcomes and exposures within our primary analysis were complete. The CEM deals with missing values by coarsening the variables as normal and uses the missing data point as a separate category. ${ }^{15}$ Therefore, these patients $(n=6)$ were eligible for the match, but none of the patients with missing data ended up in our matched cohorts. The multivariable regression models were only conducted on patients without any missing data $(n=1,617)$. Our cost analysis used complete case methods ( 35 costs outcomes were missing: ten in the no PNB group, and 25 in the PNB group).

\section{Results}

We identified 2,207 patients who underwent shoulder surgery at TOH from April 2011 to December 2016 (Fig. 1). Of those who underwent shoulder surgery, 584 patients were excluded leaving 1,623 patients eligible for our analysis. Reasons for exclusion are described in Fig. 1. Overall, PNBs were placed in 1,382 patients (85.2\%), $1,330(96.2 \%)$ of which were catheters. In the first year of the study, $21.4 \%$ of patients received no PNB for their shoulder surgery; subsequent years had a marginally greater proportion of patients who received PNB (Fig. 2).

Patient characteristics are described in Table 1. In the unmatched cohort, patients that received PNB were older, had a more recent surgery, or had rotator cuff surgery. Coarsened exact matching (one-to-many) matched 211 patients who didn't receive a PNB to 950 different patients with similar characteristics who did receive a PNB (total $n$ $=1,161 ; 71.5 \%$ of the total unmatched cohort). After matching, standardized differences within the groups was reduced to $<10 \%$ for all measured confounders. No patients died in the 30 days after surgery. In our unmatched cohort, $32(2.0 \%)$ patients had an unplanned admission after their surgery, $14(0.9 \%)$ patients were readmitted to the hospital within 30 days of their surgery, and $122(7.5 \%)$ patients were seen in the ED within 30 days of their surgery. Unadjusted and coarsened exact match-adjusted results of our primary and secondary analyses are described in Table 2.

\section{Primary outcome}

In our primary unmatched analysis, there was no difference in the risk of the composite outcome between groups (RR, 0.99; $95 \% \mathrm{CI}, 0.65$ to 1.50 ). Using CEM one-to-many adjustment, patients who received a PNB did not have a statistically different primary outcome rate compared with those who had no PNB (RR, 1.58; 95\% CI, 0.83 to 3.01). The absolute risk difference was $2.7 \%$ (95\% CI, -0.5 to 


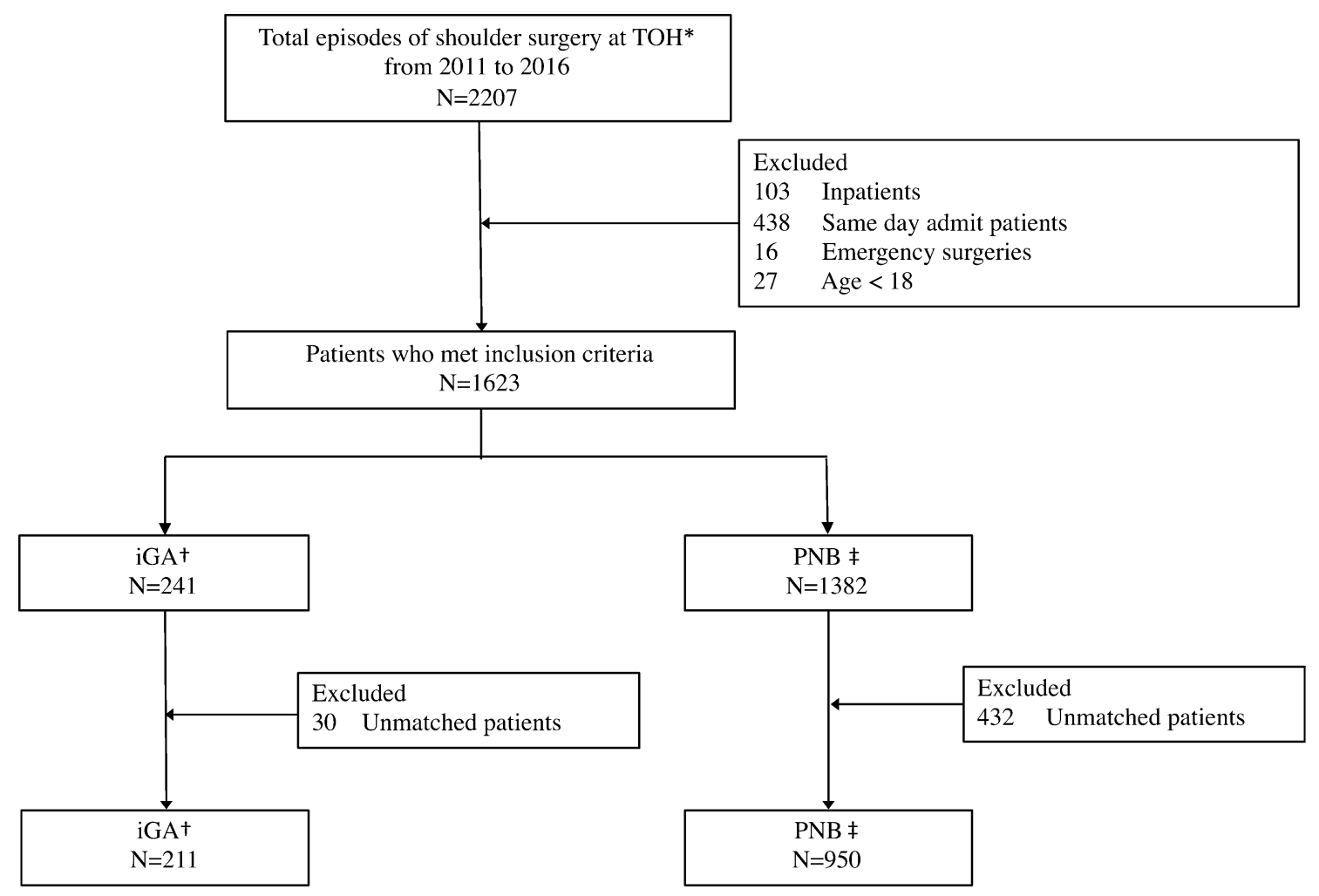

Fig. 1 Flow diagram depicting the creation of the analytical dataset. $*$ TOH $=$ The Ottawa Hospital; ${ }^{\dagger} \mathrm{iGA}=$ isolated general anesthesia; ${ }^{\star} \mathrm{PNB}=$ peripheral nerve blockade

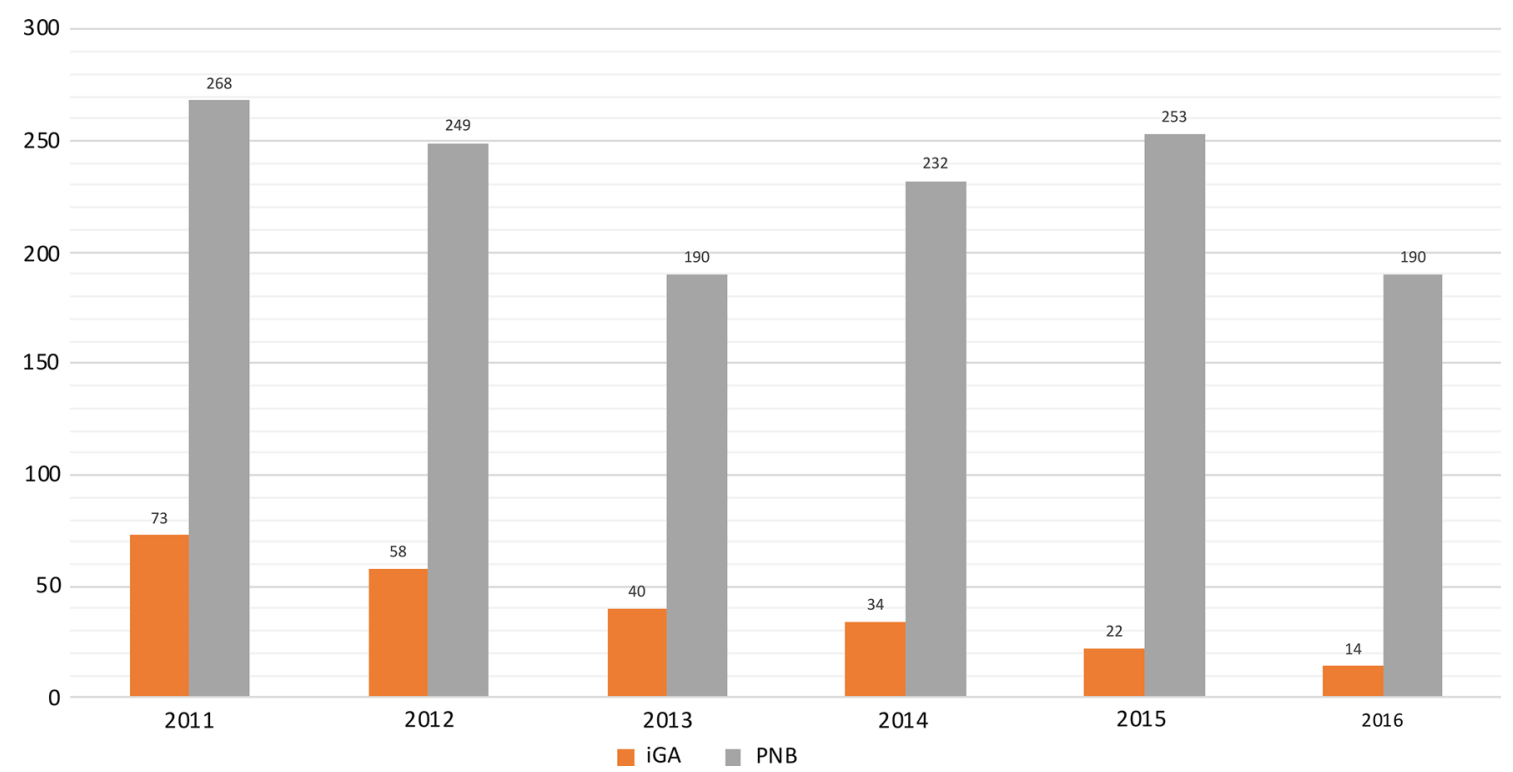

Fig. 2 Graph displaying the proportion of PNB by year of study. ${ }^{*} \mathrm{iGA}=$ isolated general anesthesia; ${ }^{\dagger}$ PNB $=$ peripheral nerve blockade

6.1). When examining the individual components of the composite outcome in the CEM one-to-many cohort, there was an increased risk of an ED visit within 30 days for those patients who received a PNB (RR, 2.84; 95\% CI, 1.14 to 7.08$)$. In the full cohort regression model, this result persisted (RR, $1.88 ; 95 \% \mathrm{CI}, 1.03$ to 3.46 ). Within this model, there was also a decreased risk of unplanned admission in those patients who received a PNB (RR, 0.37; 95\% CI, 0.18 to 0.76), which was not apparent in the CEM cohort analysis. 
Table 1 Ambulatory shoulder surgery cohort $(n=1,623)$

\begin{tabular}{|c|c|c|c|c|c|c|}
\hline & \multicolumn{3}{|l|}{ Full cohort } & \multicolumn{3}{|c|}{ After coarsened exact matching (CEM)* } \\
\hline & No PNB $(n=241)$ & $\mathrm{PNB}(n=1382)$ & SD & No PNB $(n=211) \dagger$ & PNB $(n=950)$ & SD \\
\hline Age, mean (SD) & $47.1(15.4)$ & $50.1(15.4)$ & 19.5 & $47.5(47.4)$ & $47.7(15.0)$ & 0.6 \\
\hline \multicolumn{7}{|l|}{ Sex, $n(\%)$} \\
\hline Female & $86(35.7)$ & $455(32.9)$ & 1.8 & $55.5(26.3)$ & $250(26.3)$ & 0 \\
\hline \multicolumn{7}{|c|}{ Year of surgery, $n(\%)$} \\
\hline $2011 / 12$ & $131(54.4)$ & $517(37.4)$ & 2.8 & $98.6(46.7)$ & $444(46.7)$ & 0 \\
\hline $2013 / 14$ & $74(30.7)$ & $422(30.5)$ & 0.2 & $67.5(32.0)$ & $304(32.0)$ & 0 \\
\hline $2015 / 16$ & $36(14.9)$ & $443(32.1)$ & 30.0 & $44.9(21.3)$ & $202(21.3)$ & 0 \\
\hline \multicolumn{7}{|l|}{$\mathrm{ASA}^{*}, n(\%)$} \\
\hline$\leq 2$ & $183(77.9)$ & $1054(76.3)$ & 0.5 & $177.7(84.2)$ & $800(84.2)$ & 0 \\
\hline$>2$ & $52(22.1)$ & $328(23.7)$ & 2.7 & $33.3(15.8)$ & $150(15.8)$ & 0 \\
\hline \multicolumn{7}{|c|}{ Surgery location, $n(\%)$} \\
\hline Shoulder joint & $188(78.0)$ & $852(61.7)$ & 14.4 & $152.6(72.3)$ & $687(72.3)$ & 0 \\
\hline Rotator cuff & $45(18.7)$ & $528(38.2)$ & 17.2 & $58.4(27.7)$ & $263(27.7)$ & 0 \\
\hline Shoulder muscle & $8(3.3)$ & $2(0.1)$ & 24.1 & 0 & 0 & \\
\hline \multicolumn{7}{|l|}{ Surgery type, $n(\%)$} \\
\hline Arthroscopic & $212(88.0)$ & $1297(93.8)$ & 16.6 & $206.8(98.0)$ & $931(98.0)$ & 0 \\
\hline Open & $29(12.0)$ & $85(6.2)$ & 16.6 & $4.2(2.0)$ & $19(2.0)$ & 0 \\
\hline \multicolumn{7}{|l|}{ Campus, $n(\%)$} \\
\hline Civic & $24(10.0)$ & $143(10.4)$ & 0.8 & $14.0(6.6)$ & $63(6.6)$ & 0 \\
\hline General & $129(53.5)$ & $487(35.2)$ & 4.2 & $76.6(36.3)$ & $345(36.3)$ & 0 \\
\hline Riverside & $88(36.5)$ & $752(54.4)$ & 3.3 & $120.4(57.1)$ & $542(57.1)$ & 0 \\
\hline \multicolumn{7}{|c|}{ Elixhauser comorbidity, $n(\%)$} \\
\hline$<0$ & $0(0)$ & $0(0)$ & N/A & 0 & 0 & 0 \\
\hline 0 & $240(99.6)$ & $1380(99.9)$ & 0 & $211(100)$ & $950(100)$ & \\
\hline $1-5$ & $* *$ & $0(0)$ & N/A & 0 & 0 & \\
\hline$>5$ & $0(0)$ & $* *$ & N/A & 0 & 0 & \\
\hline
\end{tabular}

*Variables included in the CEM model included age, sex, year, ASA, surgery location, surgery type, campus, Elixhauser comorbidity. **Small cell size, unable to report. $\dagger$ Note: weighted frequencies for the "no PNB" group are presented. "Six missing values for ASA score, all from the "no PNB" group

ASA = American Society of Anesthesiologists; $\mathrm{CEM}=$ coarsened exact matching; N/A = not available; PNB = peripheral nerve block; SD = standard deviation

\section{Secondary outcome}

In our secondary analysis, within the matched cohort, there was no significant difference in the mean (standard deviation [SD]) total costs incurred after surgery for patients who received a PNB compared with those patients who had no PNB (\$82 [667] vs \$60 [483], respectively; ratio of means, 0.73 ; $95 \% \mathrm{CI}, 0.21$ to 2.49 ). The cost difference attributable to a PNB was $\$ 1(95 \% \mathrm{CI}$, - 52 to 117). Of note, and as discussed in the methods, the cost of the surgery and/or PNB was not included in this cost. The $\mathrm{R}^{2}$ value for the cost model containing components of the composite outcome was 0.32 , which suggests that the majority of cost variation was not explained by the component outcomes alone.

\section{Sensitivity analyses}

Our sensitivity analyses produced similar results when compared with our primary (Table 3). The findings of our subgroup analysis are described in Table 4. Comparing the type of PNB (catheter $v s$ single shot), there was no difference in risk of our composite outcome between iGA (reference group), patients who received a catheter (RR, 1.10; 95\% CI, 0.73 to 1.67), or single shot PNB (RR, 0.76; 95\% CI, 0.27 to 2.08). In addition, when comparing the type of anesthesia (i.e., iGA, iPNB, or GA + PNB), there was no difference in the risk of composite outcome between our reference group (iGA) and the other anesthesia types. 
Table 2 Association of peripheral nerve blocks with outcomes in ambulatory shoulder surgery (primary and secondary)

\begin{tabular}{|c|c|c|c|c|}
\hline & No PNB & PNB & $\mathrm{RR}^{* *}(95 \% \mathrm{CI})$ & $P$ value \\
\hline \multicolumn{5}{|l|}{ Primary analysis } \\
\hline Full cohort (crude analysis) & $n=241$ & $n=1,382$ & & \\
\hline Composite outcome, $n(\%)$ & $23(9.5)$ & $130(9.4)$ & $0.99(0.65$ to 1.50$)$ & 0.95 \\
\hline Unplanned admission, $n(\%)$ & $12(5.0)$ & $20(1.5)$ & $0.29(0.14$ to 0.58$)$ & $0.001 *$ \\
\hline Readmission within 30 days, $n(\%)$ & $3(1.2)$ & $11(0.8)$ & $0.64(0.18$ to 2.27$)$ & 0.49 \\
\hline ED visits within 30 days, $n(\%)$ & $11(4.6)$ & $111(8.0)$ & $1.76(0.96$ to 3.22$)$ & 0.067 \\
\hline Coarsened exact match adjusted (one-to-many) $\dagger$ & $n=211^{*}$ & $n=950$ & & \\
\hline Composite outcome, $n(\%)$ & $10.0(4.7)$ & $71(7.5)$ & $1.58(0.83$ to 3.01$)$ & 0.16 \\
\hline Unplanned admission, $n(\%)$ & $5.1(2.4)$ & $9(1.0)$ & $0.39(0.13$ to 1.14$)$ & 0.08 \\
\hline Readmission within 30 days, $n(\%)$ & $0.7(0.3)$ & $5(0.5)$ & $1.67(0.13$ to 21.4$)$ & 0.70 \\
\hline ED visits within 30 days, $n(\%)$ & $4.8(2.3)$ & $62(6.5)$ & $2.84(1.14$ to 7.08$)$ & $0.025^{*}$ \\
\hline \multicolumn{5}{|l|}{ Secondary analysis ${ }^{\S \|}$} \\
\hline Unmatched cohort & $n=231$ & $n=1357$ & & \\
\hline Cost after surgery, median (IQR) & $0(0,0)$ & $0(0,0)$ & $0.44(0.14$ to 1.36$)$ & 0.15 \\
\hline Coarsened exact match adjusted (one-to-many) $\dagger$ & $n=202$ & $n=925$ & & \\
\hline Cost after surgery, median (IQR) & $0(0,0)$ & $0(0,0)$ & $0.73(0.21$ to 2.49$)$ & 0.62 \\
\hline
\end{tabular}

$* P<0.05$ is statistically significant. $†$ Variables included in the CEM model included age, sex, year, ASA, surgery location, surgery type, campus, Elixhauser comorbidity. "Note: weighted frequencies of outcomes for the "no PNB" group are presented. ${ }^{\S}$ Using a log-gamma regression model given the distribution of the cost data (right-skewed). IMissing cost data from 35 patients (10 in "no PNB" group, 25 in "PNB" group)

**RR refers to risk ratios for all outcomes, other than costs, which are presented as a ratio of means

ASA = American Society of Anesthesiologists; $\mathrm{CEM}=$ coarsened exact matching; $\mathrm{CI}=$ confidence interval; $\mathrm{ED}=$ emergency department; IQR = interquartile range; $\mathrm{PNB}=$ peripheral nerve block

\section{Discussion}

In this retrospective cohort study examining PNBs in ambulatory shoulder surgery, there was no association between anesthesia type and the composite outcome of unplanned admissions, readmissions within 30 days, or ED visits within 30 days. We also found no difference in costs. Although PNBs are known to improve immediate postoperative pain, our study provides some evidence that PNBs may in fact negatively impact post-discharge outcomes. Interestingly, our rate of postoperative adverse outcomes was substantially lower than those reported in the literature, which primarily originate from the United States. Whether this reflects a difference in health system structure and related performance requires further study.

To date, the large body of literature examining the impact of regional anesthesia on shoulder surgery outcomes has largely focused on immediate postoperative outcomes, such as acute pain scores and length of PACU stay. While these outcomes are clearly important and can substantially impact patient satisfaction and day-of-surgery discharge rates, a significant knowledge gap remains around the longer-term impact of PNBs on post-discharge outcomes. ${ }^{27,28}$ The current evidence base features a number of studies evaluating single shot PNBs and their influence on day-of-surgery discharge. ${ }^{23-26}$ Four studies described a reduced admission rate after surgery in patients with a PNB; one study found that admissions were shorter in patients with PNB compared with those without. ${ }^{23}$ Nevertheless, data describing the association of PNBs with post-discharge outcomes following ambulatory surgery are poorly described. In a single-centre randomized trial of 50 patients, there were no self-reported complications in any patients in the two weeks after surgery. ${ }^{26}$ Three retrospective studies report post-discharge outcomes. One group found no difference in 30-day mortality ( $0 \%$ in PNB group vs $0.05 \%$ in GA group) ${ }^{25}$ whereas another found that regional anesthesia (OR, $0.72 ; 99 \% \mathrm{CI}, 0.56$ to 0.92$)$ was associated with lower odds of requiring acute care (ED visits or readmissions within seven days of surgery). ${ }^{29}$ Most recently, a retrospective study examining patients undergoing total shoulder arthroplasty found that, compared with GA, the administration of RA was associated with lower rates of total in-hospital complications, reduced 90-day hospital readmissions, and greater homebound discharge. ${ }^{30}$ In contrast, in a population of patients who received wrist fracture surgery, the rate of ED visits and readmissions were higher in patients receiving single shot PNBs compared with patients receiving $\mathrm{GA}^{31}$ 
Table 3 Sensitivity analyses of the matching algorithm and composite outcome measure

\begin{tabular}{|c|c|c|c|c|}
\hline & No PNB & PNB & Risk ratio $(95 \% \mathrm{CI})$ & $P$ value \\
\hline Coarsened exact match adjusted* (one-to-one) & $n=210$ & $n=210$ & & \\
\hline Composite outcome, $\dagger(n(\%)$ & $16(7.6)$ & $16(7.6)$ & $1.00(0.51$ to 1.95$)$ & 1.0 \\
\hline Unplanned admission, $n(\%)$ & $6(2.9)$ & $4(1.9)$ & $0.67(0.19$ to 2.33$)$ & 0.53 \\
\hline Readmission within 30 days, $n(\%)$ & $2(1.0)$ & $1(0.5)$ & $0.50(0.05$ to 5.5$)$ & 0.57 \\
\hline ED visits with 30 days, $n(\%)$ & $10(4.8)$ & $12(5.7)$ & $1.2(0.53$ to 2.72$)$ & 0.66 \\
\hline \multicolumn{5}{|l|}{ Regression models } \\
\hline Full cohort $*$ & $n=235$ & $n=1,382$ & & \\
\hline Composite outcome, $n(\%)$ & $23(9.79)$ & $130(9.4)$ & $1.08(0.72$ to 1.64$)$ & 0.71 \\
\hline Unplanned admission, $n(\%)$ & $12(5.11)$ & $20(1.45)$ & $0.37(0.18$ to 0.76$)$ & $0.01^{\S}$ \\
\hline Readmission within 30 days, $n(\%)$ & $3(1.28)$ & $11(0.8)$ & $0.80(0.22$ to 2.91$)$ & 0.73 \\
\hline ED visits with 30 days, $n(\%)$ & $11(4.68)$ & $111(8.0)$ & 1.88 (1.03 to 3.46$)$ & $0.04^{\S}$ \\
\hline Coarsened exact match adjusted I (one-to-many) & $n=211$ & $n=950$ & & \\
\hline Composite outcome $(n, \%)$ & $10.0(4.7)$ & $71(7.5)$ & $1.62(0.85$ to 3.07$)$ & 0.14 \\
\hline
\end{tabular}

*Variables included in the CEM one-to-one model included age, sex, year, ASA, surgery location, surgery type, campus, Elixhauser comorbidity. †Two patients had multiple outcomes, therefore the composite outcome components add up to 18 , not 16

${ }^{*}$ Variables included in the regression model included age, sex, year, ASA, surgery location, surgery type, campus, Elixhauser comorbidity. ${ }^{\S} P<$ 0.05 is statistically significant. IAdjusted for continuous variables in a log binomial regression model (age, ASA, year, Elixhauser comorbidity). $\mathrm{ASA}=$ American Society of Anesthesiologists; $\mathrm{CEM}=$ coarsened exact matching; $\mathrm{CI}=$ confidence interval; $\mathrm{ED}=$ emergency department; $\mathrm{PNB}=$ peripheral nerve block

Table 4 Outcome sensitivity analyses using multivariable regression models

\begin{tabular}{|c|c|c|c|c|}
\hline Cohort & Outcome & Composite outcome $n(\%)$ & Risk ratio $(95 \% \mathrm{CI})^{*}$ & $P$ value \\
\hline \multirow[t]{4}{*}{ Full } & PNB type & & & \\
\hline & No PNB $(n=241)$ & $23(9.5)$ & 1 (reference) & \\
\hline & PNB (catheter) $(n=1330)$ & $126(9.5)$ & $1.10(0.73$ to 1.67$)$ & 0.65 \\
\hline & PNB (single shot) $(n=52)$ & $4(7.7)$ & $0.76(0.27$ to 2.08$)$ & 0.59 \\
\hline \multirow[t]{3}{*}{ Cohort without single shot blocks } & PNB type & & & \\
\hline & No PNB $(n=241)$ & $23(9.5)$ & 1 (reference) & \\
\hline & PNB (catheter) $(n=1330)$ & $126(9.5)$ & $1.10(0.73$ to 1.67$)$ & 0.64 \\
\hline \multirow[t]{3}{*}{ Cohort without single shot blocks or joint arthroplasties } & PNB type & & & \\
\hline & No PNB $(n=235)$ & $20(8.5)$ & 1 (reference) & \\
\hline & PNB (catheter) $(n=1,319)$ & $121(9.2)$ & $1.16(0.74$ to 1.82$)$ & 0.51 \\
\hline \multirow[t]{4}{*}{ Full } & Anesthesia type & & & \\
\hline & $\mathrm{iGA}(n=241)$ & $23(9.5)$ & 1 (reference) & \\
\hline & $\mathrm{GA}+\mathrm{PNB}(n=774)$ & $80(10.3)$ & $1.16(0.75$ to 1.79$)$ & 0.49 \\
\hline & iPNB $(n=608)$ & $50(8.22)$ & $0.98(0.61$ to 1.56$)$ & 0.92 \\
\hline
\end{tabular}

*Variables included in the adjusted model include age, sex, year, ASA, surgery location, surgery type, campus, Elixhauser comorbidity. $\dagger P<$ 0.05 is statistically significant

ASA = American Society of Anesthesiologists; CI = confidence interval; GA = general anesthesia; iGA $=$ isolated general anesthesia; $\mathrm{iPNB}=$ isolated peripheral nerve block; $\mathrm{PNB}=$ peripheral nerve block

The current study provides novel data, specific to the Canadian health care system, to enhance our understanding of the association between PNBs and longer-term postoperative outcomes. Our paper differs from other available studies in the literature in that we captured ED visits, which are common but rarely reported, and we utilized a clinically meaningful composite outcome. As ambulatory shoulder surgery is relatively low risk, the composite outcome increases the event rate to support statistical power, ${ }^{32}$ and can lead to earlier identification of 
real improvements in care. $^{33}$ Nevertheless, composite outcomes can lead to difficulties in interpreting findings, as we discuss below. Additionally, over $90 \%$ of the PNBs placed in this study were ambulatory catheters. While the analgesic benefit of a single-shot PNB is approximately 12-15 hr, once the block wears off, patients are required to use alternate analgesic strategies to manage their pain. Thus, continuous peripheral catheters may offer the benefit of extending analgesia beyond the duration of clinically important pain. Studies have not extensively examined administration of catheters $v s$ single shot PNBs in healthcare, but a number of case series have shown safety and efficacy of PNB catheters in the ambulatory setting. ${ }^{34,35}$

In defining our composite outcome, we hypothesized that the known positive impact of PNBs on day-of-surgery outcomes would translate beyond hospital discharge, especially since the majority of PNBs were placed as catheters. Nevertheless, despite the lack of significant differences in primary or secondary outcomes between people with and without PNBs, the differing directional associations found warrant closer examination. First, ED visits within 30 days, which were the most common adverse health system outcome, were found to be significantly higher in the PNB group. Whether the higher incidence of these outcomes was due to specific block-related problems is unknown, but this warrants future research as this post-discharge outcome appears to be directionally incongruent with day-of-surgery outcome. Second, unplanned day-of-surgery admissions were lower in the PNB group, not statistically significant in the CEM one-to-many model but significant in the full cohort regression. This directional signal is consistent with the literature and the presumed mechanism of improved acute pain control in people with a PNB. Lastly, readmission occurred in $0.8 \%$ and $1.2 \%$ of patients in the PNB and no PNB groups respectively. Overall, the rate of adverse outcomes was much lower than rates reported from other centres and healthcare systems. Day-of-surgery admission rates were five to ten times lower in our study than in previous studies. Thirty-day readmission rates were reported to be approximately $5 \%$ in ambulatory orthopedic surgery, ${ }^{36-38}$ compared with $1 \%$ in our study. This may partly reflect the systems-based, multidisciplinary approach instituted in our centre, which includes routine post-discharge follow up.

Another recently published study addressed the population-level impact of anesthesia care on shoulder surgery outcomes. ${ }^{30}$ Ding et al., using the New York Statewide Planning and Research Cooperative System (SPARCS) database, studied 4,158 patients undergoing shoulder arthroplasty to measure the association between anesthesia type (GA or GA plus PNB $v s$ isolated PNB) using a propensity-matched cohort analysis. They found that patients receiving GA had higher 90-day readmission rates than patients receiving isolated RA $(6.5 \%$ vs $4.2 \%$; odds ratio $1.59 ; P=0.036$ ). Nevertheless, they did not examine the risk of visits to the ED after surgery or costs. Furthermore, it is difficult to causally link outcomes 90 days after surgery to an ambulatory procedure. While their results support a positive impact of PNBs on some perioperative outcomes, this study suffered from a number of limitations. First, the SPARCS database only recorded the single highest level of anesthesia provided, thus patients who received GA would only have a code for GA, regardless of whether they received concomitant regional blockade, which would bias their exposure. Second, the authors could not be certain that all cases were planned as ambulatory cases; their sample likely included inpatients. Third, they examined only two exposure levels, GA (or GA + PNB) and iPNB; and no attempt was made to identify or assess the impact of continuous catheters or GA + PNB on outcomes.

The divergence of our findings from Ding et al. is likely multifactorial. First, the studies were conducted in different countries with different healthcare systems, and the overall rate of day-of-surgery admissions was substantially higher in the Ding et al. study. In addition, they studied a larger sample, which provided greater statistical power for finding a significant difference. Second, in our study, which covered the same time span as Ding et al., there was a significant institutional change in the way anesthesia was provided to patients (i.e., a significant increase in isolated PNB use; Fig. 2). This change in practice suggests that other unmeasured changes in practice may have occurred, which we were able to account for (at least in part) in our CEM approach, which included an exact match on year of surgery; Ding et al.'s PS-matched study did not account for the year of surgery, which could bias results in an unpredictable manner. Compared with other studies in the literature that found a reduction in the unplanned dayof-surgery admission rates in PNB patients, ${ }^{23,24,26}$ our study featured stronger control for confounding and a larger sample size. While we did find a non-significant decrease in day-of-surgery admissions, our lower than expected rate in the no PNB group may have led to type 2 errors; almost 1,300 patients per arm would be needed to detect a $50 \%$ reduction (i.e., $2 \%$ to $1 \%$ ) in this outcome with $80 \%$ power and a $5 \%$ significance level.

Within Canada, no studies have looked at long-term ambulatory shoulder surgery outcomes, such as unplanned admissions or return to the ED after surgery. One study from a Canadian institution examined the effectiveness of four anesthesia methods for arthroscopic shoulder surgery, with their primary outcome being time to discharge from the PACU. ${ }^{39}$ They found that interscalene nerve blocks 
significantly reduced PACU time (drop of $27 \mathrm{~min} ; 95 \% \mathrm{CI}$, 17.3 to 37.2), and reduced pain upon arrival to the PACU. Such findings highlight the need for future prospective randomized trials in regional anesthesia to include not only day-of-surgery outcomes, but also post-discharge patient and system outcomes.

\section{Limitations}

This study has a number of limitations. As an observational study, our findings are at risk of several types of bias. First, there is risk of exposure misclassification bias, however, we utilized the medico-legal standard for anesthesia data collection to define receipt of a PNB. Second, confounding bias may have influenced the receipt of specific anesthesia interventions and outcomes; if unmeasured confounders led people with higher risk of post-discharge adverse events to preferentially receive a block our findings would be biased to the null. Nevertheless, we did use advanced methods for observational research shown to be less biased than PS methods to control for pre-specified measured confounders. We also pre-registered our protocol, which pre-specified our outcomes and reduced the risk of type 1 errors, also known as "p-hacking" in data science. Our sensitivity analyses provide insight into the robustness of our analyses, which were consistent across approaches. Unfortunately, reasons for readmissions and visits to the ED were not available to us, and lack specificity within Canadian healthcare data. In addition, the surgical billing codes (CCI classification) lack granularity on the potential for differing postoperative pain, although we did conduct a sensitivity analysis to try to account for some of these differences. Third, while we examined important postdischarge 30-day outcomes, this study did not examine longer-term outcomes such as quality of life scores, or complications that last more than 30 days (i.e., neurologic complications, chronic pain). Cost analyses captured postoperative costs at the hospital level but were not adequately granular to account for costs of supplies in the operating room. Fourth, we do not have data that specifically link the anesthesiologist or surgeon to the patient, which is a potential unmeasured confounder. Our REB specifically excluded these identifiers in the privacy review process because of risk of re-identification. Fifth, this study examines data obtained from within a single academic health sciences centre. While these factors will limit generalizability, we would expect our findings to be applicable to similar hospitals and healthcare systems across Canada. Last, given the unexpected lower rates of adverse events in our institution, our results may in fact be due to this study being underpowered to detect a difference between the groups.
Implications

This study provides insight into the impact of anesthesia interventions on patient outcomes and health system performance in ambulatory shoulder surgery. Although PNBs are known to improve postoperative pain, our study provides some evidence that PNBs can negatively impact post-discharge outcomes after ambulatory surgery in a Canadian healthcare setting.

Acknowledgements The authors acknowledge the assistance of Sascha Davis, Information Specialist (Learning Services, The Ottawa Hospital, Ottawa, Ontario, Canada), for her assistance in developing and executing our literature search. The authors also acknowledge the Canadian Anesthesia Society for supporting this research.

Conflicts of interest None declared.

Editorial responsibility This submission was handled by Dr. Hilary P. Grocott, Editor-in-Chief, Canadian Journal of Anesthesia.

Author contributions Gavin M. Hamilton, Reva Ramlogan, and Daniel I. McIsaac contributed to all aspects of this manuscript, including study conception and design, and data acquisition, analysis, and interpretation. They also contributed to drafting the article. Anne Lui contributed to the conception and design of the study and interpretation of data. Colin J.L. McCartney and Faraj Abdallah contributed to the conception and design of the study.

Funding sources CAS Resident Research Grant. Daniel I. McIsaac receives salary support from The Ottawa Hospital Department of Anesthesiology and Pain Medicine, is a Clinical Research Chair at the University of Ottawa, and is supported by the CAS Career Scientist Award.

\section{References}

1. Cullen KA, Hall MJ, Golosinskiy A. Ambulatory surgery in the United States, 2006. Natl Heal Stat Rep 1997; 11: 1-25.

2. Jain NB, Higgins LD, Losina E, Collins J, Blazar PE, Katz JN. Epidemiology of musculoskeletal upper extremity ambulatory surgery in the United States. BMC Musculoskelet Disord 2014; 15: 4 .

3. Fortier J, Chung $F, S u ~ J$. Unanticipated admission after ambulatory surgery-a prospective study. Can J Anaesth 1998; 45: 612-9.

4. Memtsoudis SG, Ma Y, Swamidoss CP, Edwards AM, Mazumdar $M$, Liguori GA. Factors influencing unexpected disposition after orthopedic ambulatory surgery. J Clin Anesth 2012; 24: 89-95.

5. Gerbershagen HJ, Aduckathil S, van Wijck A, Peelen LM, Kalkman CJ, Meissner W. Pain intensity on the first day after surgery: a prospective cohort study comparing 179 surgical procedures. Anesthesiology 2013; 118: 934-44.

6. Wennberg J, Gittelsohn A. Small area variations in health care delivery 1973; 182: 1102-8.

7. Hughes MS, Matava MJ, Wright RW, Brophy RH, Smith $M V$. Interscalene brachial plexus block for arthroscopic shoulder 
surgery: a systematic review. J Bone Joint Surg Am 2013; 95 : 1318-24.

8. von Elm E, Altman DG, Egger $M$, et al. Strengthening the Reporting of Observational Studies in Epidemiology (STROBE) statement: guidelines for reporting observational studies. Br Med J 2007; 335: 806-8.

9. Benchimol EI, Smeeth L, Guttmann A, et al. The REporting of studies Conducted using Observational Routinely-collected health Data (RECORD) statement. PLoS Med 2015; 12: e1001885.

10. McIsaac DI, Bryson GL, van Walraven C. Impact of ambulatory surgery day of the week on postoperative outcomes: a populationbased cohort study. Can J Anesth 2015; 62: 857-65.

11. Waterman BR, Dunn JC, Bader J, Urrea L, Schoenfeld AJ, Belmont PJ. Thirty-day morbidity and mortality after elective total shoulder arthroplasty: patient-based and surgical risk factors. J Shoulder Elbow Surg 2015; 24: 24-30.

12. Wodchis W, Bushmeneva K, Nikitovic M, McKillop I. Guidelines on person level cost using administrative databases in Ontario. Working Paper Series - Volume 1, May 2013. Available from URL: http:// www.hsprn.ca/uploads/files/Guidelines_on_PersonLevel_Costing May_2013.pdf (accessed August 2018).

13. Quan H, Sundararajan V, Halfon $P$, et al. Coding algorithms for defining comorbidities in ICD-9-CM and ICD-10 administrative data. Med Care 2005; 43: 1130-9.

14. Austin PC. Using the standardized difference to compare the prevalence of a binary variable between two groups in observational research. Commun Stat Simul Comput 2009; 38: 1228-34.

15. Blackwell M, Iacus $S$, King G, Porro G. CEM: coarsened exact matching in stata. Stata J 2009; 9: 524-46.

16. Iacus SM, King G, Porro G. Multivariate matching methods that are monotonic imbalance bounding. J Am Stat Assoc 2011; 106: 345-61.

17. King G, Nielsen R, Coberley C, Pope JE, Wells A. Comparative effectiveness of matching methods for causal inference. Unpublished Manuscript 2011. Available from URL: http://j. mp/2nydGlv (accessed August 2018).

18. van Walraven C, Austin PC, Jennings A, Quan H, Forster AJ. A modification of the Elixhauser comorbidity measures into a point system for hospital death using administrative data. Med Care 2009; 47: 626-33.

19. Austin PC, Ghali WA, Tu JV. A comparison of several regression models for analysing cost of CABG surgery. Stat Med 2003; 22: 2799-815

20. Austin PC. Statistical criteria for selecting the optimal number of untreated subjects matched to each treated subject when using many-to-one matching on the propensity score. Am J Epidemiol 2010; 172: 1092-7.

21. Austin PC. A tutorial and case study in propensity score analysis: an application to estimating the effect of in-hospital smoking cessation counseling on mortality. Multivariate Behav Res 2011; 46: 119-51.

22. McNutt LA, Wu C, Xue X, Hafner JP. Estimating the relative risk in cohort studies and clinical trials of common outcomes. Am $\mathbf{J}$ Epidemiol 2003; 157: 940-3.

23. Brown AR, Weiss $R$, Greenberg $C$, Flatow EL, Bigliani $L U$. Interscalene block for shoulder arthroscop: comparison with general anesthesia. Arthroscopy 1993; 9: 295-300.

24. D'Alessio JG, Rosenblum $M$, Shea $K P$, Freitas DG. A retrospective comparison of interscalene block and general anesthesia for ambulatory surgery shoulder arthroscopy. Reg Anesth 1995; 20: 62-8.

25. Danninger T, Stundner $O$, Rasul $R$, et al. Factors associated with hospital admission after rotator cuff repair: the role of peripheral nerve blockade. J Clin Anesth 2015; 27: 566-73.

26. Hadzic A, Williams BA, Karaca PE, et al. For outpatient rotator cuff surgery, nerve block anesthesia provides superior same-day recovery over general anesthesia. Anesthesiology 2005; 102: $1001-7$.

27. Atchabahian A, Schwartz G, Hall CB, Lajam CM, Andreae MH. Regional analgesia for improvement of long-term functional outcome after elective large joint replacement. Cochrane Database Syst Rev 2015; 8: CD010278.

28. Warrender WJ, Syed UA, Hammoud S, et al. Pain management after outpatient shoulder arthroscopy: a systematic review of randomized controlled trials. Am J Sports Med 2017; 45: 167686.

29. Liu J, Flynn DN, Liu WM, Fleisher LA, Elkassabany NM. Hospital-based acute care within 7 days of discharge after outpatient arthroscopic shoulder surgery. Anesth Analg 2018; 126: 600-5.

30. Ding DY, Mahure SA, Mollon B, Shamah SD, Zuckerman JD, Kwon $Y W$. Comparison of general versus isolated regional anesthesia in total shoulder arthroplasty: a retrospective propensity-matched cohort analysis. J Orthop 2017; 14: 417-24.

31. Sunderland S, Yarnold CH, Head SJ, et al. Regional versus general anesthesia and the incidence of unplanned health care resource utilization for postoperative pain after wrist fracture surgery: results from a retrospective quality improvement project. Reg Anesth Pain Med 2016; 41: 22-7.

32. Jammer I, Wickboldt $N$, Sander M, et al. Standards for definitions and use of outcome measures for clinical effectiveness research in perioperative medicine: European Perioperative Clinical Outcome (EPCO) definitions: a statement from the ESAESICM joint taskforce on perioperative outcome measures. Eur J Anaesthesiol 2015; 32: 88-105.

33. Myles PS, Devereaux PJ. Pros and cons of composite endpoints in anesthesia trials. Anesthesiology 2010; 113: 776-8.

34. Marhofer P, Anderl W, Heuberer $P$, et al. A retrospective analysis of 509 consecutive interscalene catheter insertions for ambulatory surgery. Anaesthesia 2015; 70: 41-6.

35. Fredrickson MJ, Leightley $P$, Wong A, Chaddock M, Abeysekera $A$, Frampton $C$. An analysis of 1505 consecutive patients receiving continuous interscalene analgesia at home: a multicentre prospective safety study. Anaesthesia 2016; 71: 373-9.

36. Bernatz JT, Tueting JL, Anderson PA. Thirty-day readmission rates in orthopedics: a systematic review and meta-analysis. PLoS One 2015; 10: e0123593.

37. Mahoney A, Bosco JA 3rd, Zuckerman JD. Readmission after shoulder arthroplasty. J Shoulder Elbow Surg 2014; 23: 377-81.

38. Sherman SL, Lyman S, Koulouvaris $P$, Willis A, Marx RG. Risk factors for readmission and revision surgery following rotator cuff repair. Clin Orthop Relat Res 2008; 466: 608-13.

39. Bosco L, Zhou C, Murdoch JA, et al. Pre- or postoperative interscalene block and/or general anesthesia for arthroscopic shoulder surgery: a retrospective observational study. Can J Anesth 2017; 64: 1048-58. 